\section{Internação hospitalar e mortalidade por esquistossomose mansônica no Estado de Pernambuco, Brasil, 1992/2000}

\author{
Hospitalization and mortality from mansoni \\ schistosomiasis in the State of Pernambuco, \\ Brazil, 1992/2000
}

Ana Paula da Costa Resendes 1

Reinaldo Souza-Santos 1

Constança Simões Barbosa ${ }^{2}$

\footnotetext{
1 Escola Nacional de Saúde Pública, Fundação Oswaldo Cruz, Rio de Janeiro, Brasil.

2 Centro de Pesquisas Aggeu Magalhães, Fundação Oswaldo Cruz, Recife, Brasil.

Correspondência R. Souza-Santos

Departamento de Endemias Samuel Pessoa, Escola Nacional de Saúde Pública, Fundação Oswaldo Cruz, Rua Leopoldo Bulhões 1480, Rio de Janeiro, $R J$ 21041-210, Brasil. rssantos@ensp.fiocruz.br
}

\begin{abstract}
In order to investigate the historical trends, epidemiological profile, and spatial distribution of hospital admissions and deaths from schistosomiasis in the State of Pernambuco, Brazil, an analysis was conducted of data from the Hospital Information System and Mortality Information System from 1992 to 2000. The results showed a reduction in hospital admissions and mortality, while identifying more admissions and deaths among males. There was a lower percentage of deaths and admissions from schistosomiasis in individuals under 30 years of age. However, schistosomiasis is still of relevant magnitude, as evidenced by the number of deaths from this cause and the number of patients admitted to the hospital system in Pernambuco. A spatial analysis of the endemic's distribution in the State showed that although from 1995 to 1999 there was a greater spread of admissions due to schistosomiasis in the municipalities (counties) of the Sertão (backlands) and São Francisco river valley, the number of municipalities with hospitalizations due to schistosomiasis decreased from 1995 to 1998, followed by an increase in 1999 and 2000.
\end{abstract}

Indicators of Morbidity and Mortality; Mortality; Schistosomiasis; Spatial Analysis

\section{Introdução}

O controle da esquistossomose é uma das tarefas mais difíceis dos serviços de saúde pública em razão da ampla difusão dos hospedeiros intermediários, dos mecanismos de escape com relação à existência de métodos de controle, da freqüência do contato humano com a água em atividades agrícolas, doméstica e/ou por lazer, das dinâmicas próprias de cada microfoco de transmissão, da falta de água potável, das limitações do tratamento individual e em massa e da falta de abordagem preventiva associada à curativa na organização dos serviços 1 .

A identificação das áreas geográficas e grupos populacionais que apresentam maior risco de adoecimento e morte constitui uma tarefa imprescindível para a elaboração de programas preventivos e como meio de avaliação de exposições diferenciadas 2 . O reconhecimento desses grupos supõe, por sua vez, a seleção de intervenções sociais e sanitárias para diminuir ou eliminar os fatores específicos de risco, sugerindo um processo de diagnóstico, ação, avaliação e adequação das estratégias de atuação dos serviços de saúde ${ }^{3}$.

A relevância da esquistossomose como problema de Saúde Pública não se restringe à sua antiguidade e ampla distribuição geográfica mundial. Um dos fatores diz respeito à relevância econômica, expressa pelo impacto na atividade produtiva da população infectada. A ocor- 
rência de manifestações clínicas graves em indivíduos infectados pelo Schistosoma mansoni tem sido considerada um dos aspectos mais importantes desta parasitose, tornando-a objeto de atuação por parte de programas de controle em países endêmicos 4 .

A endemia apresenta ampla distribuição no Brasil, devendo-se destacar que a transmissão ocorre de forma mais intensa nas áreas litorâneas e da mata de alguns Estados nordestinos, particularmente Alagoas, Pernambuco, Sergipe e Bahia, além do norte de Minas Gerais. Estas duas áreas da Região Nordeste, área litorânea e da mata, reúnem condições ecológicas e de organização do espaço que propiciam intensa transmissão 5 . Entre essas condições favoráveis destaca-se a existência da Biomphalaria glabrata, considerado o mais eficiente hospedeiro intermediário, e a escassez de serviços de saneamento.

O Estado de Pernambuco, mesmo com todos os esforços da Fundação Nacional de Saúde (FUNASA), apresentou no início da década de 90 taxas de infecção humana crescentes, passando por situações de prevalência crônicas, na região da Zona da Mata, com até $80,0 \%$ dos indivíduos parasitados em certas localidades 6, ou, ainda, casos de infecção aguda freqüentes, como os que vêm ocorrendo no litoral do Estado 7. Os moluscos, hospedeiros intermediários, são da espécie $B$. glabrata, com ocorrência em focos isolados no litoral do Estado, e B. straminea que tem ampla distribuição na Zona de Mata podendo ser encontrada até os limites da região agreste 8 .

Em estudo recente para o Estado de Pernambuco, Carmo ${ }^{4}$ verificou uma redução da taxa de mortalidade de 3,75 em 1979 para 2,04 por 100 mil habitantes em 1996 e queda da proporção de internações por esquistossomose, em relação ao total de internações, de 6,07 em 1984 para 4,51 (10 mil internações) em 1998. Entretanto, a prevalência por esquistossomose no período de 1983 a 1995 foi superior a 30,0\% em alguns municípios do Estado de Pernambuco 4 .

O presente trabalho tem como objetivo realizar uma análise da internação hospitalar e mortalidade por esquistossomose em Pernambuco no período de 1992 a 2000 e descrever a distribuição espacial da endemia no Estado, de 1995 a 2000.

\section{Metodologia}

O Estado de Pernambuco está situado na parte centro-leste da Região Nordeste do Brasil. Apresenta área de $98.281 \mathrm{~km}^{2} \mathrm{e}$ uma população de 7.918.344 habitantes. O clima é tropical e úmido no litoral e semi-árido no agreste e sertão. Na maior parte do território, a temperatura média varia de $16^{\circ}$ a $31^{\circ} \mathrm{C}$. Possui 184 municípios e o território de Fernando de Noronha. A pluviosidade, que cai acentuadamente do litoral para o interior, mantém-se sempre abaixo de $600 \mathrm{~mm}$ anuais. Apresenta-se dividido em cinco regiões (Metropolitana, Zona da Mata, Agreste, Sertão e São Francisco) com diversas características climáticas e fisiográficas (http:// www.pe.gov.br).

Os dados de internação hospitalar e óbitos por esquistossomose, para o período de 1992/ 2000, foram obtidos na base de dados do Sistema de Informações Hospitalares (SIH) e do Sistema de Informações sobre Mortalidade (SIM), do Ministério da Saúde. Foram consideradas as causas básicas referidas como esquistossomose (CID 09: capítulo 1 - 072; e CID10: capítulo 1 - B65). Dados anteriores ao ano de 1992 não se encontram estratificados por sexo e faixa etária nessas bases, devido a isso, não foi possível trabalhar uma série mais extensa.

Foram calculados o coeficiente de internação hospitalar e o coeficiente de mortalidade por esquistossomose por 100 mil habitantes, o percentual de internação hospitalar e o percentual de mortalidade por esquistossomose em relação às doenças infecciosas e parasitárias por 100 mil habitantes, o coeficiente de internação hospitalar e mortalidade por esquistossomose segundo sexo e faixa etária e a proporção de internação hospitalar em relação ao total de internações (por 10 mil internações). Como denominador do coeficiente de internação hospitalar e mortalidade, utilizaram-se dados populacionais dos censos de 1991 e 2000, contagem 1996 e projeções intercensitárias (http:// www.ibge.gov.br).

Como indicador de morbidade hospitalar para a construção da série histórica, foi utilizada a proporção de internações por esquistossomose em relação ao total de internações (para cada 10 mil internações). Esse indicador foi inicialmente utilizado por Carmo 4 , sendo preferível em relação ao coeficiente de internação, no qual é utilizado como denominador a população, que não evidencia a variabilidade anual no total de internações. 
Para verificar a distribuição espacial da endemia no Estado foi calculado o coeficiente de internação hospitalar utilizando o município de residência como unidade de análise. O período analisado foi de 1995 a 2000, uma vez que estes dados estão disponíveis apenas a partir de 1995. Como a Zona da Mata é endêmica para esquistossomose e apresenta os maiores coeficientes de internação hospitalar, no período estudado e em relação a todo o Estado, a área foi escolhida para uma análise mais detalhada. Assim, foi calculada a prevalência de período das internações hospitalares por 100 mil habitantes para a Zona da Mata. A análise espacial foi realizada por intermédio de mapas temáticos gerados no programa MapInfo. O coeficiente de internação hospitalar em Pernambuco foi estratificado em 5 faixas: sem internações; de 0,10 a 9,99; de 10,00 a 19,99; de 20,00 a 29,99; e de 30,00 a 120,00 internações por 100 mil habitantes. A prevalência de período das internações hospitalares para os 43 municípios da região da Zona da Mata foi calculada dividindo-se a soma do número de casos de internação por esquistossomose, no período de 1995/2000, pela média populacional para o mesmo período. A prevalência de período foi estratificada em 5 faixas: sem informações; de 1 a 50; de 51 a 100; de 101 a 150; e de 151 a 200 por 100 mil habitantes.

\section{Resultados}

Foi verificado um total de 3.342 internações por esquistossomose em Pernambuco no período de 1992 a 2000. A análise do coeficiente de internação hospitalar (por 100 mil habitantes) por esquistossomose mostra uma redução de 7,48 em 1992 para 3,46 em 1998, voltando a aumentar nos dois últimos anos. Os resultados evidenciam um aumento do coeficiente de mortalidade (por 100 mil habitantes) de 2,19 em 1992 para 2,89 em 1995, uma redução entre 1996 e 1998 e novamente um aumento em 1999 e 2000 , com coeficiente de 1,78 e 1,98 , respectivamente (Tabela 1 ).

A distribuição por idade mostra que a internação hospitalar por esquistossomose é um acontecimento importante em todas as faixas etárias. No grupo de 10 20 anos ocorreram $14,63 \%$ das internações, o que contribuiu para que o percentual acumulado em menores de 20 anos tenha alcançado $20,68 \%$. O maior número de internações ocorreu na faixa de 60 anos e mais, com percentual de $27,68 \%$. Os óbitos por esquistossomose são eventos raros em menores de 20 anos, com aproximadamente 1,28\% do total conhecido. Já no grupo de 40 a 50 anos de idade ocorreram $14,11 \%$ dos óbitos, fato que contribui para que o percentual acumulado para menores de 50 anos tenha alcançado $25,73 \%$. O maior número de óbitos ocorreu na faixa etária de 60 anos e mais, com percentual de 51,18\% (Tabela 2).

O coeficiente de internação hospitalar por esquistossomose, segundo faixa etária e sexo, mostra valores ligeiramente maiores nos indivíduos do sexo masculino em praticamente todas as faixas etárias estudadas. Foi verificado um aumento do coeficiente de internação hospitalar com o avanço da idade. A análise do coeficiente de mortalidade por esquistossomose segundo faixa etária e sexo indica, também, número ligeiramente maior de óbitos em indivíduos do sexo masculino e um aumento desse coeficiente de acordo a idade (Tabela 3 ).

A análise da proporção de internações por esquistossomose em relação ao total de internações por doenças infecciosas e parasitárias não mostra uma tendência. Um total de 26.223 óbitos por doenças infecciosas e parasitárias (Capítulo 1 da CID-09 e da CID-10) foi registrado no Estado, no período de 1992 a 2000, sendo 1.467 (5,59\%) devido à esquistossomose. Apesar da redução no número de internações por doenças infecciosas e parasitárias no período, de 90.256 em 1992 para 47.133 em 2000, observou-se um aumento na proporção de internações por esquistossomose em relação ao total de internações por doenças infecciosas e parasitárias, de 0,59 em 1992 para 0,74 em 2000. A mortalidade proporcional por esquistossomose no grupo das doenças infecciosas e parasitárias e o total de óbitos no grupo das doenças infecciosas e parasitárias não seguem uma tendência. Se compararmos os anos de 1992 e 2000, houve um pequeno aumento da mortalidade proporcional de 5,32 em 1992 para 6,06 em 2000.

A proporção de internações por esquistossomose em relação ao total de internações (10 mil internações) revela uma redução de 6,87 em 1992 para 4,83 em 1996, aumentando para 6,21 em 2000. Houve uma redução do total de internações de 781.827 em 1992 para 560.306 em 2000 (Tabela 4).

Os mapas da taxa de internação hospitalar por esquistossomose segundo os municípios de Pernambuco, para o período de 1995/2000, estão dispostos na Figura 1. Pode-se observar para todos os anos que as taxas mais elevadas estão localizadas na região da Zona da Mata. Embora tenha ocorrido, entre 1995 e 1999, uma maior dispersão das internações por esquistossomose para os municípios do Sertão e São Francisco, o número de municípios com internação hospitalar esquistossomose sofreu uma 
redução em todo o Estado entre 1995 e 1998, seguido de um aumento para o ano de 1999 e 2000. Comparando os anos de 1995 e 2000, o número de municípios com internação em razão da esquistossomose foi aproximadamente o mesmo: 68 municípios para o ano de 1995 e 67 para o ano de 2000.

A análise da região da Zona da Mata não mostra uma tendência temporal. Ocorreu um aumento do número de municípios com internação por esquistossomose de 1995 (27 municípios) para 1996 (30 municípios), seguido de redução até 1999, com somente 16 municípios, e novo aumento em 2000 (29 municípios).

Quanto à prevalência de período de internações hospitalares na região da Zona da Mata (Figura 2), observa-se a presença de um aglomerado com taxas maiores na parte norte da região, composto por aproximadamente $14 \mathrm{mu}$ nicípios. Verifica-se que apenas os municípios de Primavera, Xexéu e Jaqueira, localizados na parte sul da região, não apresentaram internações por esquistossomose. Os municípios de Timbaúba, Nazaré da Mata e Rio Formoso apresentaram taxas de prevalência de período de internações hospitalares por 100 mil habitantes na faixa de 151 a 200 casos; para os municípios de Vicência e Aliança, as taxas se encontram na faixa de 101 a 150 . Dentre esses municípios, somente Rio Formoso se localiza na porção sul da Zona da Mata.

\section{Discussão}

Na análise de indicadores de morbi-mortalidade emerge discussão sobre a validade das informações para descrever a ocorrência de um problema de saúde em determinada população. Entre os indicadores utilizados no presente estudo, o interesse maior recai sobre o índice de internação hospitalar, à medida que, acerca dos indicadores de mortalidade, existe um maior conhecimento dos dados e fontes de informações disponíveis hoje no país 9 .

Um aspecto importante que deve ser considerado é que a análise dos dados de internação hospitalar por esquistossomose, realizada no presente estudo nos oferece uma estimativa da ocorrência das formas graves da doença na população, especialmente da forma hepatoesplênica (HEME), à proporção que os pacientes com HEME demandam atendimento hospitalar.

Tabela 1

Número de internações hospitalares e óbitos por esquistossomose e coeficiente de internação hospitalar e mortalidade por esquistossomose (por $100 \mathrm{mil}$ habitantes), no período de 1992/2000. Estado de Pernambuco, Brasil.

\begin{tabular}{lcccc}
\hline Ano & $\begin{array}{c}\text { Internação hospitalar } \\
\mathrm{n}\end{array}$ & $\begin{array}{c}\text { Mortalidade } \\
\text { Coeficiente }\end{array}$ & $\mathrm{n}$ & \begin{tabular}{c} 
Coeficiente \\
\hline 1992
\end{tabular} \\
\hline 538 & 7,48 & 155 & 2,19 \\
1993 & 520 & 7,11 & 186 & 2,58 \\
1994 & 396 & 5,36 & 190 & 2,60 \\
1995 & 333 & 4,46 & 213 & 2,89 \\
1996 & 300 & 4,04 & 149 & 2,04 \\
1997 & 311 & 4,17 & 156 & 2,10 \\
1998 & 260 & 3,46 & 128 & 1,71 \\
1999 & 336 & 4,43 & 134 & 1,78 \\
2000 & 348 & 4,39 & 156 & 1,98 \\
Total & 3.342 & 5,17 & 1.467 & 2,21 \\
\hline
\end{tabular}

Número de internações e óbitos por esquistossomose, percentual e percentual acumulado por faixa etária. Estado de Pernambuco, Brasil, 1992/2000.

\begin{tabular}{|c|c|c|c|c|c|c|}
\hline \multirow[t]{2}{*}{ Faixa etária (anos) } & \multicolumn{3}{|c|}{ Internações } & \multicolumn{3}{|c|}{ Óbitos } \\
\hline & $\mathrm{n}$ & $\%$ & $\%$ acumulado & $\mathrm{n}$ & $\%$ & $\%$ acumulado \\
\hline $0 \vdash 10$ & 202 & 6,04 & - & 04 & 0,27 & - \\
\hline $10 \vdash 20$ & 489 & 14,63 & 20,68 & 15 & 1,01 & 1,28 \\
\hline $20 \vdash 30$ & 249 & 7,45 & 28,13 & 48 & 3,24 & 4,52 \\
\hline $30 \vdash 40$ & 413 & 12,36 & 40,48 & 105 & 7,09 & 11,61 \\
\hline $40 \vdash 50$ & 509 & 15,23 & 55,72 & 209 & 14,11 & 25,73 \\
\hline $50 \vdash 60$ & 549 & 16,43 & 72,14 & 328 & 22,15 & 47,87 \\
\hline 60 e mais & 925 & 27,68 & 99,82 & 758 & 51,18 & 99,05 \\
\hline Idade ignorada & 6 & 0,18 & 100,00 & 14 & 0,95 & 100,00 \\
\hline Total & 3.342 & & & 1.481 & & \\
\hline
\end{tabular}


1396 Resendes APC et al.

Tabela 3

Coeficiente de internação hospitalar e mortalidade por esquistossomose (100 mil habitantes), segundo faixa etária e sexo, no período de 1992/2000. Estado de Pernambuco, Brasil.

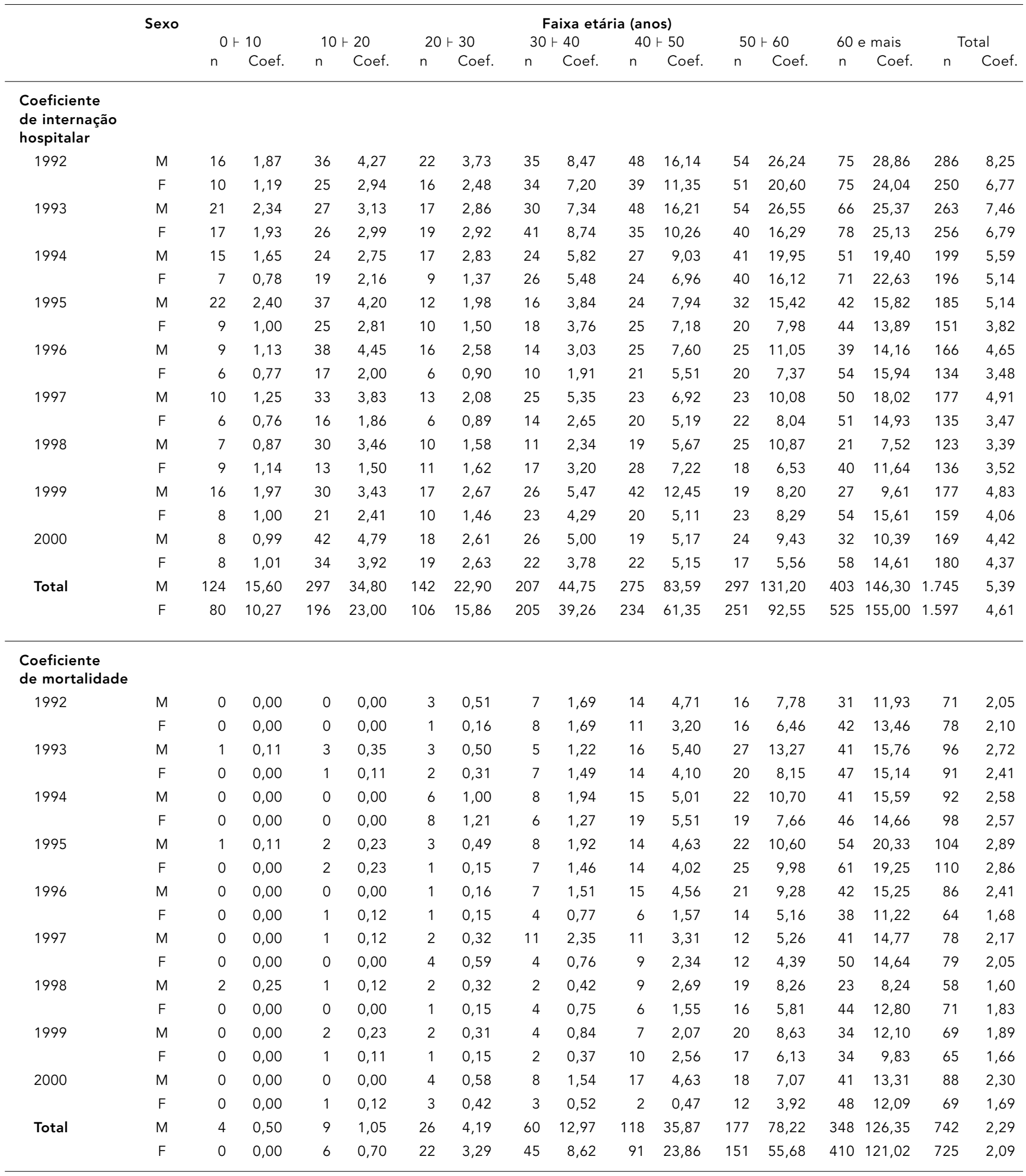

Cad. Saúde Pública, Rio de Janeiro, 21(5):1392-1401, set-out, 2005 
É importante, contudo, ressaltar que alguns fatores contribuem para a subestimativa do número de casos de HEME em uma população, com utilização de dados hospitalares. Muitas vezes a condição diagnosticada não corresponde necessariamente à doença ou agravo, mas inclui síndromes, sinais ou sintomas, conforme estabelecido pela Classificação Internacional de Doença (CID), com isso, alguns pacientes com HEME são internados com diagnósticos associados à doença. Outro fator relevante está relacionado à demanda e à oferta de serviços hospitalares. Alguns pacientes com essa patologia, demandando assistência hospitalar, não são internados por restrição de oferta de leitos ou de serviços diagnósticos e terapêuticos, custeados pelo Sistema Único de Saúde 4. Outro problema reside no fato de que o nível sócio-econômico está relacionado ao acesso à hospitalização. Espera-se que indivíduos com baixo nível sócio-econômico tenham menos chances de dar entrada em centros de referência, pois a internação nestes serviços depende da distância entre a moradia e o hospital, conhecimentos e indicações médicas 10. Todavia, a utilização de dados do SIH constitui instrumento significativo para o conhecimento da ocorrência dessa enfermidade na população. Ademais, representa a única fonte disponível de informações para o estudo de formas graves da esquistossomose, sobretudo a HEME.

A decisão em utilizar os dados de internação hospitalar foi decorrente da impossibilidade de acesso aos registros dos inquéritos parasitológicos desenvolvidos nos municípios, única forma de medir a ocorrência da doença. Ainda que os registros de internação hospitalar e mortalidade não sejam os mais adequados para a compreensão da dinâmica de transmissão da esquistossomose, pode-se ter uma visão aproximada da gravidade da doença.

Os resultados aqui encontrados apontam um número ligeiramente maior de internações e óbitos em indivíduos do sexo masculino para todas as faixas etárias estudadas e em praticamente todos os anos, indicando um padrão de exposição. Homens e mulheres, cada qual a seu tempo e modo, vivem experiências específicas e privadas e não compartilhadas pelo sexo oposto. Sob o ponto de vista epidemiológico, esta diversidade biológica e social, implica disparidade quanto à exposição a riscos.

A associação entre sexo e infecção é muitas vezes contraditória, e o seu valor em predizer o risco de infecção é ineficiente. Em geral, atividades ocupacionais, econômicas, entre outras, são mais úteis como indicadores de risco do que simplesmente o sexo ${ }^{11}$. Devemos lembrar
Tabela 4

Proporção de internações por esquistossomose em relação ao total de internações (10 mil internações), no período de 1992/2000. Estado de Pernambuco, Brasil.

\begin{tabular}{lcc}
\hline Ano & $\begin{array}{c}\text { Proporção } \\
\text { esquistossomose }\end{array}$ & $\begin{array}{c}\text { Total de } \\
\text { internações }\end{array}$ \\
\hline 1992 & 6,87 & 781.827 \\
1993 & 6,84 & 758.912 \\
1994 & 5,37 & 735.785 \\
1995 & 5,29 & 627.697 \\
1996 & 4,83 & 619.312 \\
1997 & 5,16 & 602.265 \\
1998 & 4,49 & 579.234 \\
1999 & 5,81 & 578.774 \\
2000 & 6,21 & 560.306 \\
\hline
\end{tabular}

que algumas atividades são desenvolvidas predominantemente por indivíduos do sexo masculino ou feminino.

Em estudo realizado nas comunidades de Itapinassu, onde a principal atividade ocupacional é a agricultura, e de São Joaquim, onde a principal atividade ocupacional é a cerâmica, ambas no Município de Tracunhaém, Estado de Pernambuco, foi verificada associação estatisticamente significante entre a prevalência da esquistossomose e a variável sexo, sendo a positividade maior em indivíduos do sexo masculino. Houve maior intensidade de infecção e formas severas em Itapinassu; porém, não foram estatisticamente significantes. Isso pode ser explicado pelas diferenças na ocupação resultando em uma maior exposição dos agricultores 12 .

Um outro aspecto que merece discussão é a análise da mortalidade e internação hospitalar segundo faixa etária. Ainda que represente uma série temporal pequena, o menor número, em termos percentuais, de óbitos e de pacientes internados por esquistossomose em menores de trinta anos pode ser atribuído ao impacto da quimioterapia no tratamento seletivo do grupo de 2 a 14 anos, adotada como medida de controle nos municípios com prevalência entre $25,0 \%$ e 50,0\% no período de 1980 a 198613 .

Além disso, os percentuais de internações e óbitos por esquistossomose, maiores nas faixas etárias mais avançadas, provavelmente, devemse a aspectos imunológicos e fisiológicos característicos da idade e ao aspecto crônico da doença.

A análise da tendência do coeficiente de internação hospitalar e da proporção de internações por esquistossomose em relação ao total de internações mostra redução desses indica- 
Figura 1

Coeficiente de internação hospitalar por esquistossomose, segundo município de residência (100 mil habitantes).

Municípios do Estado de Pernambuco, Brasil, período 1995/2000.

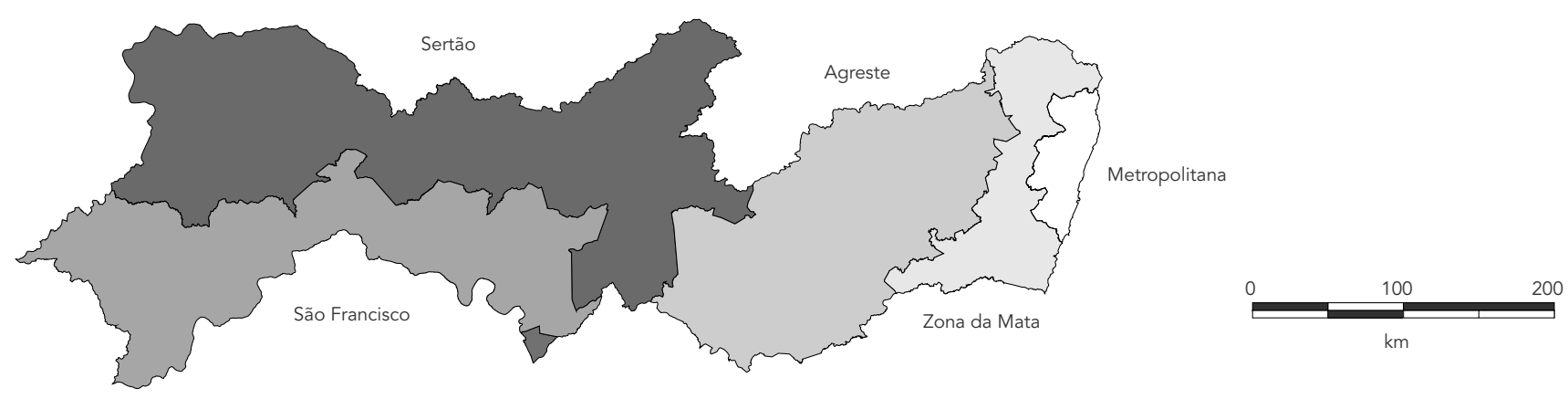

1995

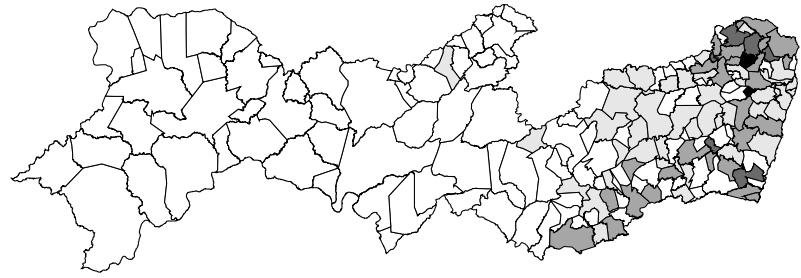

1997
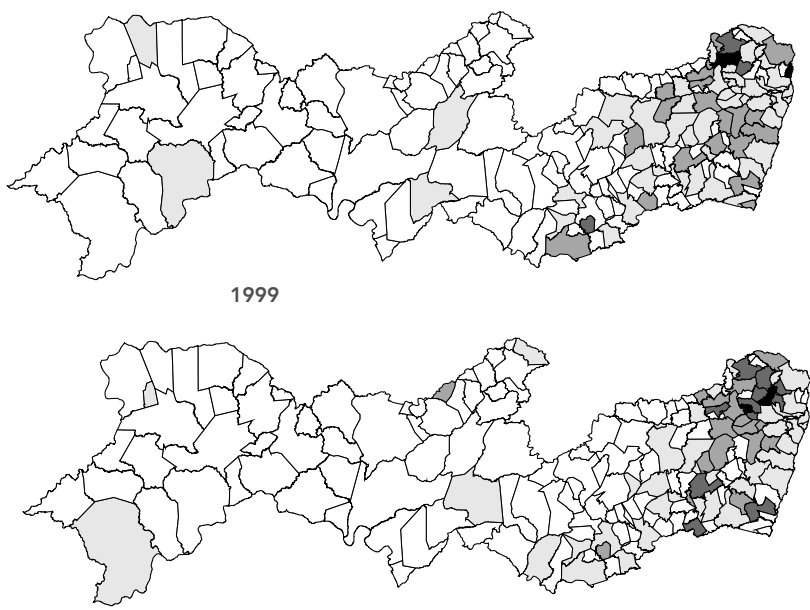

Coeficiente de internação hospitalar

30,00 a 120,00

20,00 a 29,99

10,00 a 19,99

0,10 a 9,99

sem internações

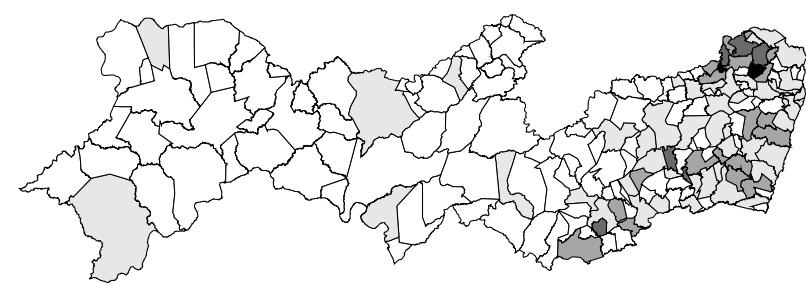

1998

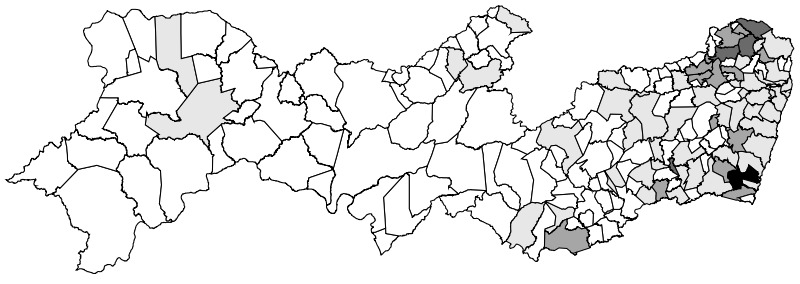

2000
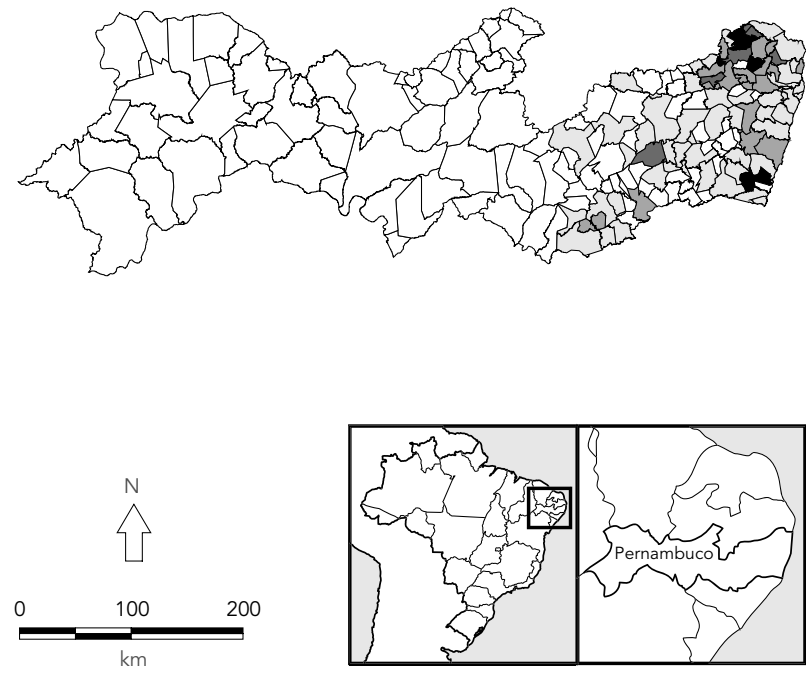
Prevalência de período de internações hospitalares por esquistossomose. Região da Zona da Mata,

Pernambuco, Brasil, período 1995/2000.

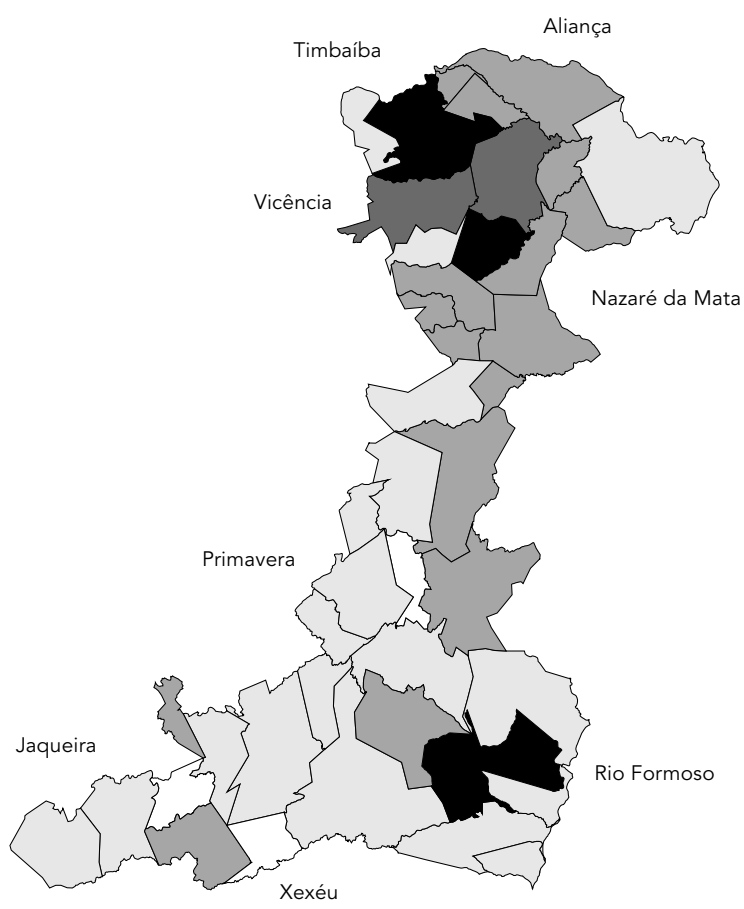

Prevalência de período/100 mil habitantes

150,00 a 200,00

100,00 a 149,99

50,00 a 99,99

1,00 a 49,99

sem internações

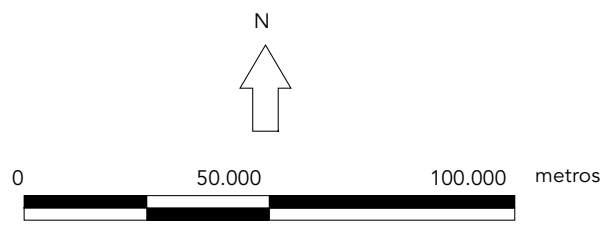

dores; entretanto, a tendência não se mantém durante todo o período de observação. Verifica-se uma reversão desta tendência nos dois últimos anos da série de estudo. O número de municípios com registros de internação hospitalar por esquistossomose, verificados por intermédio da análise espacial, mostra este mesmo padrão para Pernambuco e particularmente para a Zona da Mata. Estes achados podem apontar não só uma melhoria no sistema de informação nos dois últimos anos da série, mas também uma falha no programa de controle da endemia no Estado. Já para a mortalidade não foi observado o mesmo padrão.

Este estudo não pretende avaliar o programa de controle da esquistossomose no Estado de Pernambuco; no entanto, a experiência em diferentes Estados demonstra a necessidade de definição de estratégias de controle integradas. A quimioterapia utilizada em articulação com medidas sociais, sanitárias e ações educativas são necessárias e possibilitariam o aumento da efetividade do controle da transmissão da esquistossomose. Os programas de controle da endemia, mesmo instrumentalizados com tecnologias eficazes, não têm conseguido eficiência nas suas ações porque são pensados valendo-se de uma lógica centralizada, intervencionista, medicalizada e padronizada para todas as áreas endêmicas 14 .

Para uma mesma localidade, município ou região, a intensidade de infecção e a ocorrência de formas graves não representam um processo estático, mas podem ser continuamente modificadas por mecanismos relacionados a fatores ambientais e individuais. No contexto populacional entre os fatores que têm sido mais associados à modificação na infecção e morbidade por esquistossomose, destacamos as melhorias nas condições de abastecimento de água e saneamento básico em uma vertente, e a realização de quimioterapia na população em outra. Ambos os fatores tendem a reduzir a prevalência e a intensidade de infecção e a ocorrência de formas graves 4 .

Neste estudo, pode-se notar que a distribuição da prevalência de período das internações hospitalares por esquistossomose na região da 
Zona da Mata não ocorre de forma homogênea, existindo um aglomerado de municípios com taxas elevadas na parte norte dessa região. Para melhor compreensão deste cenário é fundamental um estudo mais amplo com introdução de outras variáveis relacionadas às condições de vida e saúde das populações e variáveis ligadas a aspectos ambientais e comportamentais. Deve-se lembrar que fatores ambientais e individuais favorecem ou inibem a intensidade de infecção e a ocorrência de formas graves. Além disso, a variação de fatores climáticos e ecológicos pode influenciar a dinâmica da população dos caramujos transmissores. É importante ressaltar que a não-ocorrência de internações por esquistossomose nos municípios de Primavera, Xexéu e Jaqueira pode ser devida a subnotificação dos casos.

A ocorrência e distribuição da esquistossomose estão ligadas a uma cadeia ampla e complexa de determinação, caracterizada pela contribuição de aspectos do meio físico e social. Salienta-se que as práticas culturais e comportamentais dos seres humanos frente às coleções hídricas são também importantes. Os elementos do meio físico podem constituir as condições ecológicas favoráveis ao desenvolvimento da doença; porém, ela só se manifesta quando combinada com aspectos sócio-culturais. Isso decorre da forma como o homem, organizado socialmente, se apropria do espaço natural, inscrevendo, no tempo, traços humanos. A forma como se darão as relações sociais definirá o espaço construído no território, o que viabilizará ou não a ocorrência da doença 15 e interferirá em sua distribuição.

O Estado de Pernambuco, inclusive a região da Zona da Mata, apresenta condições de sa- neamento básico precárias. Esse fator aliado a aspectos ligados ao comportamento populacional, a condições ambientais propícias à existência do hospedeiro intermediário, à intensa mobilidade das comunidades e à falta de um programa de controle eficaz, que depende de cada prefeitura, tudo concorre para a criação de condições propícias à manutenção da transmissão e à expansão da esquistossomose, fazendo com que Pernambuco seja o segundo Estado da Região Nordeste com as maiores taxas de prevalência.

Segundo Merchan-Hamann 16, existe uma grande heterogeneidade nos programas de controle desenvolvidos para cada município, há falta de uniformidade de ações, gerando variedade nos intervalos entre os inquéritos e, conseqüentemente, entre os ciclos de quimioterapia; além de deficiência de pessoal e de infraestrutura para execução das medidas de controle, determinando com isso uma enorme dificuldade na análise de dados de esquistossomose no país.

Os resultados aqui encontrados confirmam que a esquistossomose ainda é responsável por internações e óbitos em Pernambuco, sobretudo em áreas onde é indiscutível a necessidade de melhoria das condições de vida da população humana.

Para finalizar, vale dizer que é necessário haver mais discussões acerca da melhoria na qualidade dos registros dos sistemas de informação em saúde a fim de que as informações geradas sejam valiosas para ações de controle. Contudo, a utilização de dados do SIH já constitui instrumento valioso para o conhecimento da ocorrência e da gravidade dessa enfermidade na população.

\section{Resumo}

Com o objetivo de conhecer as tendências históricas, o perfil epidemiológico e a distribuição espacial das internações hospitalares e óbitos por esquistossomose no Estado de Pernambuco, Brasil, procedeu-se à análise de dados do Sistema de Informações Hospitalares e do Sistema de Informações sobre Mortalidade entre 1992 e 2000. Os resultados evidenciaram uma redução das internações e mortalidade, além de apontar maior número de internações e óbitos em indivíduos do sexo masculino. Houve menor percentual de óbitos e de pacientes internados por esquistossomose em menores de 30 anos. No entanto, a esquistossomose ainda apresenta uma importante magnitude evidenciada pelo número de óbitos por esta causa e pelo número de pa- cientes internados na rede hospitalar em Pernambuco. Por meio da análise espacial da distribuição da endemia no Estado, pode-se verificar que, apesar de ter ocorrido entre 1995 e 1999 uma maior dispersão das internações por esquistossomose para os municípios do Sertão e São Francisco, o número de municípios com internação hospitalar por causa da esquistossomose sofreu uma redução para Pernambuco entre 1995 e 1998, seguido de um aumento para o ano de 1999 e 2000.

Indicadores de Morbi-mortalidade; Mortalidade; Esquistossomose; Análise Espacial 


\section{Colaboradores}

Todos os autores participaram da concepção do artigo, análise e interpretação dos dados, além de lerem e aprovarem a versão final.

\section{Referências}

1. Coura-Filho P. Participação popular no controle da esquistossomose através do Sistema Único de Saúde (SUS), em Taquaraçu de Minas, (Minas Gerais, Brasil), entre 1985-1995: construção de um modelo alternativo. Cad Saúde Pública 1998; 14 Suppl 2:111-22.

2. Barcellos C, Coutinho K, Pina MF, Magalhães MMAF, Paola JCMD, Santos SM. Inter-relacionamento de dados ambientáis e de saúde: análise de risco à saúde aplicada ao abastecimento de água no Rio de Janeiro utilizando Sistemas de Informações Geográficas. Cad Saúde Pública 1998; 14:597-605.

3. Organização Pan-Americana da Saúde. Sistemas de Informação Geográfica aplicados à epidemiologia. In: Sistemas de Informação Geográfica em saúde: conceitos básicos. Brasília: Organização Pan-Americana da Saúde; 2000. p. 11-43.

4. Carmo EH. Morbidade e mortalidade por esquistossomose mansônica na Região Nordeste do Brasil [Tese de Doutorado]. Salvador: Instituto de Saúde Coletiva, Universidade Federal da Bahia; 1999.

5. Silva LJ. A esquistossomose mansônica no Estado de São Paulo: origens, distribuição, epidemiologia e controle [Tese de Livre-docência]. Campinas: Faculdade de Ciências Médicas, Universidade Estadual de Campinas; 1992.

6. Barbosa CS, Silva CB. Epidemiologia da esquistossomose mansônica no engenho Bela Rosa, $\mathrm{Mu}$ nicípio de São Lourenço da Mata, PE. Cad Saúde Pública 1992; 8:83-7.

7. Barbosa CS, Domingues ALC, Abath F, Montenegro SML, Guida U, Carneiro J, et al. Epidemia de esquistossomose aguda na praia de Porto de Galinhas, Pernambuco, Brasil. Cad Saúde Pública 2001; 17:725-8.

8. Gazin P, Barbosa CS, Bouvy M, Audry P. Registro de ocorrência de vetores da esquistossomose mansônica em açude do Sertão de Pernambuco. Rev Soc Bras Med Trop 2000; 33:407-8.
9. Oliveira H, Pereira IPA. Estatísticas de mortalidade e nascidos vivos: considerações sobre principais problemas. Inf Epidemiol SUS 1997; 6:15-9.

10. Werneck GL, Almeida LM. Validade em estudos epidemiológicos. In: Medronho RA, Carvalho DM, Bloch KV, Luiz RR, Werneck GL, organizadores. Epidemiologia. São Paulo: Editora Atheneu; 2002: p. 199-213.

11. Huang Y, Manderson L. Schistosomiasis and the social patterning of infection. Acta Trop 1992; 51:175-94.

12. Coutinho EM, Abath FGC. Barbosa CS, Domingues ALC, Melo MCV, Montenegro SML, et al. Factors involved in Schistosoma mansoni infection in Rural Areas of Northeast Brazil. Mem Inst Oswaldo Cruz 1997; 92:707-15.

13. Favre TC, Pieri OS, Barbosa CS, Becl L. Avaliação das ações de controle da esquistossomose implementadas entre 1977 e 1996 na área endêmica de Pernambuco, Brasil. Rev Soc Bras Med Trop 2001; 34:569-76.

14. Barbosa CS, Silva CB, Barbosa FS. Esquistossomose: reprodução e expansão da endemia no Estado de Pernambuco no Brasil. Rev Saúde Pública 1996; 30:609-16.

15. Barreto ML. Esquistossomose mansônica: distribuição da doença e organização social do espaço [Dissertação de Mestrado]. Salvador: Departamento de Medicina Preventiva, Universidade Federal da Bahia; 1982.

16. Merchan-Hamann E. Diagnóstico macrorregional da situação das endemias das regiões Norte e Nordeste. Inf Epidemiol SUS 1997; 6:43-114.

Recebido em 24/Nov/2004

Versão final reapresentada em 04/Abr/2005 Aprovado em 08/Abr/2005 